\title{
Altered regional homogeneity of spontaneous brain activity in idiopathic trigeminal neuralgia
}

\author{
This article was published in the following Dove Press journal: \\ Neuropsychiatric Disease and Treatment \\ 13 October 2015 \\ Number of times this article has been viewed
}

\author{
Yanping Wang ${ }^{1,2}$ \\ Xiaoling Zhang ${ }^{2}$ \\ Qiaobing Guan² \\ Lihong Wan ${ }^{2}$ \\ Yahui $\mathrm{Yi}^{2}$ \\ Chun-Feng Liu' \\ 'Department of Neurology, The \\ Second Affiliated Hospital of Soochow \\ University, Suzhou, Jiangsu Province, \\ ${ }^{2}$ Department of Neurology, The \\ Second Hospital of Jiaxing City, \\ Jiaxing, Zhejiang Province, People's \\ Republic of China
}

\begin{abstract}
The pathophysiology of idiopathic trigeminal neuralgia (ITN) has conventionally been thought to be induced by neurovascular compression theory. Recent structural brain imaging evidence has suggested an additional central component for ITN pathophysiology. However, far less attention has been given to investigations of the basis of abnormal resting-state brain activity in these patients. The objective of this study was to investigate local brain activity in patients with ITN and its correlation with clinical variables of pain. Resting-state functional magnetic resonance imaging data from 17 patients with ITN and 19 age- and sex-matched healthy controls were analyzed using regional homogeneity ( $\mathrm{ReHo}$ ) analysis, which is a data-driven approach used to measure the regional synchronization of spontaneous brain activity. Patients with ITN had decreased ReHo in the left amygdala, right parahippocampal gyrus, and left cerebellum and increased ReHo in the right inferior temporal gyrus, right thalamus, right inferior parietal lobule, and left postcentral gyrus (corrected). Furthermore, the increase in ReHo in the left precentral gyrus was positively correlated with visual analog scale $(r=0.54 ; P=0.002)$. Our study found abnormal functional homogeneity of intrinsic brain activity in several regions in ITN, suggesting the maladaptivity of the process of daily pain attacks and a central role for the pathophysiology of ITN.
\end{abstract}

Keywords: trigeminal neuralgia, resting fMRI, brain, chronic pain, local connectivity

\section{Introduction}

Idiopathic trigeminal neuralgia (ITN) is characterized by paroxysmal, electric, lancinating pains, and a variety of sensory experiences. ${ }^{1,2}$ The annual incidence is 4-5 in 100,000 , and it is estimated to affect one in $15,000-20,000$ people worldwide. ${ }^{3-5}$ With the expansion of the aging population, the proportion of elderly patients with ITN has gradually increased. Despite its prevalence and potential medical and social burdens, the pathogenesis underlying these pains remains poorly understood.

The prevailing pathophysiology of trigeminal neuralgia emphasizes its peripheral nociception and central component mechanisms. Peripheral nociception describes the hyperexcitability of the nerve-vessel conflict, whereas the central component relates to central facilitation and results in central hyperexcitability of the trigeminal system that is initiated or sustained by peripheral mechanisms. ${ }^{6,7}$ An ambiguous relationship between these two mechanisms may exist, given that ITN patients endure typical paroxysmal neuralgic perception and concomitant dull background pain between the two attacks that cause the ITN patient's brain to frequently reconfigure its intrinsic activity and to adapt its functional architecture following daily attacks. It is therefore reasonable to expect these patients to have an altered brain intrinsic activity.

Over the past two decades, neuroimaging research has improved our understanding of how chronic pain (eg, migraines, arthritis, fibromyalgia, chronic back pain, and visceralgia) can affect brain structure and functions. ${ }^{8-10}$ Of particular interest,
Correspondence: Chun-Feng Liu

Department of Neurology, The Second Affiliated Hospital of Soochow University, 1055 Sanxiang Road, Suzhou 215004, Jiangsu Province, People's Republic of China

Tel +865I267783307

Email liucf@suda.edu.cn 
magnetic resonance imaging (MRI)-based brain imaging offers a suitable avenue for measuring the functional and structural alterations that underlie pain perception and chronification. ${ }^{10-12}$ Recent studies have found that ITN is associated with structural changes in pain, attention, and motor processing areas. Evidence from structural MRI studies on ITN patients have found that ITN is associated with cortical and subcortical gray matter atrophy in the somatosensory, orbitofrontal, insular, anterior cingulate, and dorsolateral prefrontal cortices in addition to the thalamus and cerebellum. ${ }^{13-16}$ In brain diffusion tensor imaging studies, lower fractional anisotropy and increased radial diffusivity, mean diffusivity, and axial diffusivity have been recorded in brain white matter including the corpus callosum, cingulum, posterior corona radiata, and superior longitudinal fasciculus of ITN patients. ${ }^{13}$ Furthermore, abnormal thalamic metabolism of $N$-acetylaspartate/creatine has been reported in ITN patients. ${ }^{17,18}$ Despite an increasing amount of research regarding the effects of ITN on brain structure and metabolism, little is known about the effects of ITN on spontaneous brain activity.

Regional homogeneity (ReHo) analysis is a data-driven method of resting-state functional MRI (rsfMRI) that can measure the similarity across a time series of a given voxel with its nearest neighbors, without a priori knowledge of the experimental design. ${ }^{19}$ ReHo analysis is primarily focused on an activity pattern within a specific brain region and has been widely used in various clinical populations and phenotypic variables to reveal the regional synchronization of spontaneous brain activity. ${ }^{20,21}$

In this study, we hypothesized that ITN patients would show altered ReHo across central pain processing areas. Our aim was 1) to detect ReHo changes in ITN patients to localize specific brain regions that may be related to the development and persistence of this debilitating facial pain and 2) to correlate clinical variables of ITN with the ReHo of each patient within significant group differences.

\section{Materials and methods}

Informed consent was obtained from all participants. The study was conducted in accordance with the Declaration of Helsinki. The study protocol was approved by the Institutional Review Board of the Second Hospital of Jiaxing City.

\section{Participants}

Seventeen ITN patients (seven males/ten females, age $=63.41 \pm 7.25$ years old) were recruited prospectively from the Department of Neurology at the Second Hospital of Jiaxing City between February 2014 and March 2015.
The inclusive criteria for the ITN patients were as follows: 1) unilateral pain in the distribution of one or more branches (the ophthalmic [V1], the maxillary [V2], and the mandibular [V3]) of the trigeminal nerve; 2) stereotyped attacks, and characteristic (intense, sharp, superficial, or stabbing paroxysmal) pains precipitated from trigger areas or by trigger factors for each patient; 3) no additional neurological or sensory deficits in all patients; and 4) no previous surgical or other invasive procedures for ITN.

For each patient, demographic and clinical details were obtained via retrospective chart reviews, and the diagnosis of ITN was confirmed by experienced neurologists (Yanping Wang and Xiaoling Zhang) according to the International Classification of Headache Disorders-2nd edition (ICHD-II). ${ }^{22}$ The exclusion criteria were 1) patients with neural-associated diseases or chronic pain other than ITN, hypertension, diabetes, or a history of brain surgery and 2) patients unable to undergo MRI scanning because of metal implantation or those with a history of claustrophobia or other psychological disorders. In addition, we also recruited 19 age- and sex-matched healthy subjects, with no family history of neuralgia, no history of substance abuse, chronic pain, systemic, psychiatric, or neurological disorders as controls. All of the participants were right handed according to their self-report. Table 1 lists the demographic information of the ITN patients and the controls.

\section{Questionnaires}

Before MRI scanning, the following questionnaires were given to the ITN patients who were asked to respond based on his or her current pain. The duration of pains (year), pain days per month (times), as well as visual analog scales (VAS) for pain was determined by asking the patient to mark the pain severity on a $10 \mathrm{~cm}$ line. Additionally, all subjects completed the depression scores Beck's Depression Inventory-II (BDI-II), ${ }^{23}$ the Hamilton Depression (HAMD)/

Table I Subject characteristics

\begin{tabular}{llll}
\hline & ITN & Controls & P-value \\
\hline Age (years old) & $63.4 I \pm 7.25$ & $62.53 \pm 7.4 I$ & 0.72 \\
Sex (M/F) & $7 / 10$ & $9 / 10$ & $>0.99$ \\
Handedness & $I 7 R$ & $I 9 R$ & $>0.99$ \\
MMSE & $28.9 \pm I .7$ & $28.1 \pm I .3$ & 0.105 \\
BDI-II & $2.4 \pm I .8$ & $2.2 \pm I .4$ & 0.67 \\
HAMD & $2.9 \pm 2.1$ & $I .9 \pm I . I$ & 0.147 \\
HAMA & $3.5 \pm 2.9$ & $\mathrm{I} .7 \pm 0.9$ & $0.035^{*}$ \\
Duration & $6.98 \pm 5.64 \mathrm{y}$, mid $5.00 \mathrm{y}$ & $\mathrm{NA}$ & $\mathrm{NA}$ \\
\hline
\end{tabular}

Note: $* p<0.05$

Abbreviations: ITN, idiopathic trigeminal neuralgia; $M / F$, male/female; $R$, right; y, year; MMSE, Mini-Mental State Examination; BDI-II, Beck's Depression Inventory-II; HAMA, Hamilton Anxiety Rating Scale; HAMD, Hamilton Depression Rating Scale; NA, not applicable. 
Anxiety (HAMA) Rating Scale, ${ }^{24}$ and the Mini-Mental State Examination. Details are presented in Table 1.

\section{MRI data acquisition}

MRI data were acquired on a 1.5 T GE Signa Excite scanner (GE Medical Systems, Milwaukee, WI, USA) using a standard GE whole-head coil. Each subject laid supine, with the head in a neutral position and fixed comfortably by a belt and foam pads during the test. High-resolution T1-weighted anatomical datasets were obtained parallel to the AC-PC plane and covering the whole brain using three-dimensional spoiled-gradient recall (SPGR) sequence (repetition time [TR]/echo time [TE]/ inversion time $[\mathrm{TI}]=10.68 \mathrm{~ms} / 4.87 \mathrm{~ms} / 380 \mathrm{~ms}$, flip angle $=15^{\circ}$, field of view $=256 \mathrm{~mm}, 140$ axial slices, voxel size $1 \times 1 \times 1$ $\left.\mathrm{mm}^{3}\right)$. Resting-state functional datasets were recorded using a T2*-weighted gradient echo spiral pulse sequence (30 axial slices, thickness $/$ gap $=5.0 / 1 \mathrm{~mm}$, in-plane resolution $=64 \times 64$, $\mathrm{TR}=2,000 \mathrm{~ms}, \mathrm{TE}=40 \mathrm{~ms}$, flip angle $=90^{\circ}$, field of view $(\mathrm{FOV})=240 \mathrm{~mm} \times 240 \mathrm{~mm})$. The scan time for the rsfMRI session for each participant was approximately 7 minutes.

\section{Data processing}

rsfMRI data were preprocessed and analyzed using Data Processing Assistant for Resting-State fMRI (DPARSF, http://www.restfmri.net), which is based on Statistical Parametric Mapping (SPM8, http://www.fil.ion.ucl.ac.uk/ spm) and Matlab. For the rsfMRI data for each subject, the first ten volumes were discarded to avoid the possible effects of scanner instability and the adaptation of subjects to the environment, and the remaining 190 volumes were corrected for timing offsets. The resultant images were then realigned and coregistered to the T1 structural image. The T1 structural image was spatially normalized to the Montreal Neurological Institute space using diffeomorphic anatomical registration through exponentiated lie algebra, a more sophisticated registration algorithm, which can achieve a more accurate inter-subject registration, realignment of small deformations, and better spatial normalization. ${ }^{25}$ The resulting parameter was used to normalize the functional images with voxel size $3 \times 3 \times 3 \mathrm{~mm}^{3}$. Temporal filter $(0.01-0.08 \mathrm{~Hz})$ was conducted to lessen low-frequency drifts and high-frequency physiological noise. Nuisance regression was performed using white matter, cerebrospinal fluid, and the six head motion parameters as covariates. No participant had head motion with $>2.0 \mathrm{~mm}$ maximum displacement or $2.0^{\circ}$ of any angular motion.

The ReHo was calculated as previously described. ${ }^{26} \mathrm{ReHo}$ evaluates the degree of local synchronization among fMRI time courses. ReHo is computed as Kendall's coefficient of concordance $(\mathrm{KCC})$ value of the ranked time series of a given voxel to its nearest neighbors in a voxel-wise way:

$$
\mathrm{W}=\frac{\sum\left(\mathrm{R}_{\mathrm{i}}\right)^{2}-\mathrm{n}(\overline{\mathrm{R}})^{2}}{\frac{1}{12} \mathrm{~K}^{2}\left(\mathrm{n}^{3}-\mathrm{n}\right)} \text {, where } \overline{\mathrm{R}}=\frac{1}{\mathrm{n}} \sum_{\mathrm{i}=1}^{\mathrm{n}} \mathrm{R}_{\mathrm{i}}
$$

where $\mathrm{W}$ is the $\mathrm{KCC}$ among time series of given voxels, the range is $0-1 ; \mathrm{R}_{\mathrm{i}}$ is the sum rank of the $i$ th time point, $\mathrm{K}$ is the number of time series within a measured cluster (here $\mathrm{K}=27$, one center voxel plus the number of its neighbors), and $n$ is the number of ranks (here, $n=190$ time points). The individual ReHo map was obtained for each subject by calculating the $\mathrm{KCC}$ value within its whole brain voxels. These intracranial voxels were extracted to make a brain mask. Each individual ReHo map was converted to a $z$-score map by subtracting the global mean ReHo and dividing the group standard deviation within the brain mask. Finally, the $z$-score maps were smoothed with a $6 \mathrm{~mm}$ full-width at half-maximum Gaussian kernel.

\section{Statistical analysis}

Demographic and clinical data were analyzed with SPSS 16.0 (SPSS Inc., Chicago, IL, USA) and ReHo of rsfMRI data with SPM8. Voxel-wise ReHo differences between the ITN patient group $(n=17)$ and the control group $(n=19)$ were performed with independent two-sample $t$-test. The threshold was set at voxel-level $P<0.01$ and cluster-level $P<0.05$, Gaussian random field correction.

\section{Results}

\section{Demographic and clinical data}

Table 1 shows the demographic information of the ITN and control groups. Table 2 demonstrates the clinical features of the ITN patients. Between-group $t$-tests identified significant group differences in HAMA, with patients of the ITN group scoring higher in anxiety (HAMA, $P<0.05$ ). The two groups displayed no differences in age, sex, handedness, MiniMental State Examination, BDI, or HDMA.

\section{Voxel-wise comparison of ReHo}

Table 3 and Figure 1 show the group differences in ReHo between the ITN group and the control group (voxel-level $P<0.01$, cluster-level $P<0.05$, Gaussian random field correction). Compared with the healthy controls, ITN patients showed decreased ReHo in the left amygdala, right parahippocampal gyrus, and left cerebellum. Increased ReHo was observed in the right inferior temporal gyrus, right 
Table 2 Subject characteristics

\begin{tabular}{|c|c|c|c|c|c|c|c|}
\hline Patient & Sex & $\begin{array}{l}\text { Age, } \\
\text { years }\end{array}$ & $\begin{array}{l}\text { Duration, } \\
\text { years }\end{array}$ & $\begin{array}{l}\text { Pain } \\
\text { location }\end{array}$ & $\begin{array}{l}\text { Pain days } \\
\text { per month }\end{array}$ & $\begin{array}{l}\text { Pain intensity } \\
\text { (VAS) }\end{array}$ & $\begin{array}{l}\text { Vessel-nerve } \\
\text { contact on MRI }\end{array}$ \\
\hline Sub0I & $M$ & 46 & 20 & V2 & 15 & 6 & $\mathrm{R}$ \\
\hline Sub02 & $\mathrm{F}$ & 69 & 10 & $\mathrm{~V} 2$ & 10 & 7 & L \\
\hline Sub03 & $\mathrm{F}$ & 56 & 3 & $\mathrm{VI}+\mathrm{V} 2$ & 10 & 5 & $\mathrm{R}$ \\
\hline Sub04 & $M$ & 58 & 6 & $\mathrm{~V} 2+\mathrm{V} 3$ & 8 & 8 & L \\
\hline Sub05 & $M$ & 60 & 1 & V2 & 6 & 5 & $\mathrm{R}$ \\
\hline Sub06 & $\mathrm{F}$ & 70 & 3 & V2 & 7 & 7 & $\mathrm{R}$ \\
\hline Sub07 & $\mathrm{F}$ & 68 & 10 & V3 & 5 & 7 & L \\
\hline Sub08 & $M$ & 69 & 5 & $\mathrm{~V} 1+\mathrm{V} 2$ & 8 & 4 & $\mathrm{R}$ \\
\hline Sub09 & $M$ & 64 & I & $\mathrm{V} 2$ & 11 & 8 & L \\
\hline Sublo & $\mathrm{F}$ & 68 & 2.5 & V3 & 15 & 7 & $\mathrm{R}$ \\
\hline SublI & $\mathrm{F}$ & 63 & 3 & $\mathrm{~V} 2+\mathrm{V} 3$ & 9 & 8 & L \\
\hline Subl2 & $\mathrm{F}$ & 63 & 20 & $\mathrm{~V} 2+\mathrm{V} 3$ & 8 & 6 & $\mathrm{R}$ \\
\hline Subl 3 & $\mathrm{~F}$ & 75 & 3 & V3 & 6 & 6 & $\mathrm{R}$ \\
\hline Subl4 & $M$ & 58 & 10 & V2 & 4 & 3 & $R$ \\
\hline Subl5 & $\mathrm{F}$ & 55 & 10 & V3 & 3 & 7 & $\mathrm{R}$ \\
\hline Subl6 & $\mathrm{F}$ & 65 & 3 & V2 & 6 & 6 & $\mathrm{R}$ \\
\hline Subl7 & $M$ & 71 & 5 & V2 & 7 & 4 & $\mathrm{R}$ \\
\hline
\end{tabular}

Note: VI, V2, V3 is the first, second and the third branch of the trigeminal nerve, respectively.

Abbreviations: M, male; F, female; VAS, visual analog scale; MRI, magnetic resonance imaging; L, left; R, right.

thalamus, right inferior parietal lobule, the left pre- and postcentral gyrus (Table 3 and Figure 1). In order to investigate the impact of anxiety-related effects on the ReHo results, we further regressed out the HAMA score of each region showing a significant between-group ReHo difference; we found that the between-group differences in ReHo were not substantially affected by the HAMA regression.

\section{Correlations between ReHo and clinical variables}

Regional correlation analyses were conducted between the clinical variables of ITN patients and the cluster mean $z$ score of each patient within the mask of significant group differences. The increase in ReHo in the left precentral gyrus was positively

Table 3 Brain areas with ReHo differences between the ITN and control groups

\begin{tabular}{|c|c|c|c|c|c|c|c|}
\hline \multirow[t]{2}{*}{ Brain regions } & \multicolumn{3}{|c|}{$\begin{array}{l}\text { MNI } \\
\text { coordinates }\end{array}$} & \multirow[t]{2}{*}{ BA } & \multirow[t]{2}{*}{ L/R } & \multirow{2}{*}{$\begin{array}{l}\text { Number } \\
\text { of } \\
\text { Voxels }\end{array}$} & \multirow[t]{2}{*}{$t$-value } \\
\hline & $\bar{x}$ & $y$ & $\mathbf{z}$ & & & & \\
\hline Inferior ten & 51 & -57 & -15 & 37 & $\mathrm{R}$ & 71 & 3.848 \\
\hline Amygdala & -12 & 3 & -30 & 28 & $\mathrm{~L}$ & 82 & -4.2591 \\
\hline Cerebellum & -9 & -87 & -21 & 18 & $\mathrm{~L}$ & 48 & -3.883 \\
\hline Parahippocampal gyrus & 12 & -12 & -21 & 34 & $\mathrm{R}$ & 42 & -3.9466 \\
\hline Thalamus & 6 & -15 & 6 & - & $\mathrm{R}$ & 43 & 4.0861 \\
\hline Inferior parietal lobule & 39 & -45 & 42 & 40 & $\mathrm{R}$ & 71 & 4.1893 \\
\hline Postcentral gyrus & -21 & -51 & 69 & 7 & $\mathrm{~L}$ & $12 \mid$ & 4.8598 \\
\hline
\end{tabular}

Notes: Coordinates $x, y, z(m m)$ are given in standard stereotactic MNI space. All regions listed are statistically significant at $P<0.05$, AlphaSim corrected.

Abbreviations: ReHo, regional homogeneity; ITN, idiopathic trigeminal neuralgia; MNI, Montreal Neurological Institute; BA, Brodmann area; L, left; R, right. correlated with VAS score $(r=0.54 ; P=0.002$, Figure 1$)$. No other significant correlations were identified between the altered ReHo and the other clinical scores assessed.

\section{Discussion}

In this study, we assessed the local synchronization of resting-state fMRI signals in patients with ITN, a unique neuropathic pain that is called the most painful condition in humans. Altered ReHo was detected in ITN patients, who showed decreased ReHo in the limbic system and cerebellum and increased ReHo in the right inferior temporal gyrus, right thalamus, right inferior parietal lobule, and the left postcentral gyrus. More importantly, increased ReHo of the left postcentral gyrus was associated with self-reported pain intensity (VAS). This study highlights the impairment to local synchronization of pain perception, pain modulation, and motor-related brain systems that is associated with central pain processing in patients with ITN.

Neural homogeneity is considered an important and robust feature of brain activity at microscale (ie, single neuron $)^{27}$ and a reliable measure of connectomics at macroscale (ie, voxel/parcellation). ${ }^{27,28}$ Several studies have suggested that ReHo is linked to various phenotypic variables (eg, age, sex, intelligence, and personality), and differs between clinical populations. More recently, Jiang et $\mathrm{al}^{21}$ and Jiang and $\mathrm{ZuO}^{29}$ systematically reviewed the regional (short-distance) functional interactions of the organizational principles of human brain function, and proposed that ReHo may stand as a neurobiologically meaningful network centrality of the local features of the brain connectome. 
A

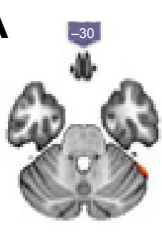

$\mathbf{L}$

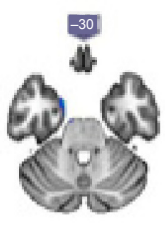


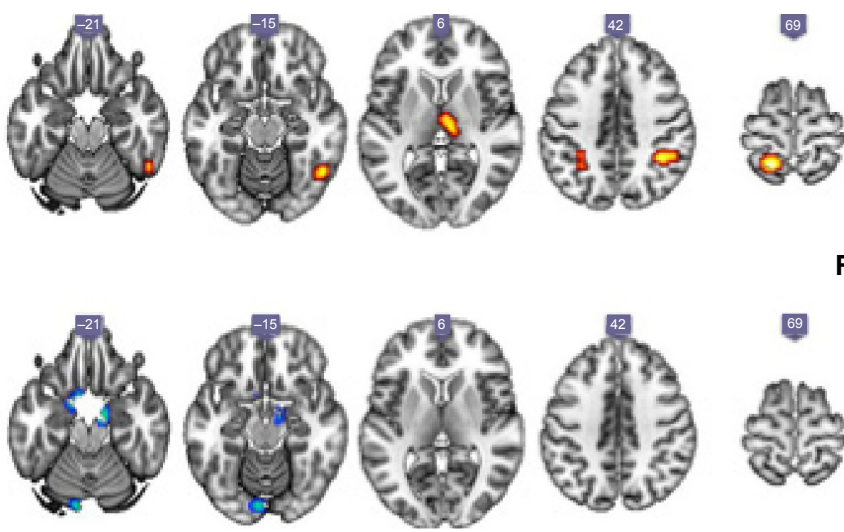

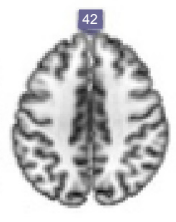

$\mathbf{R}$

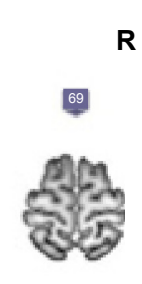

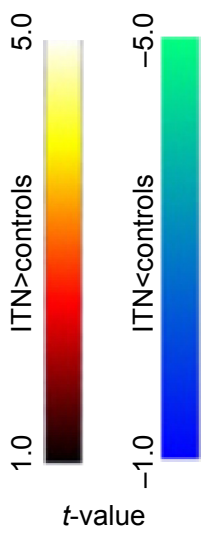

B

Plot of the significant clusters

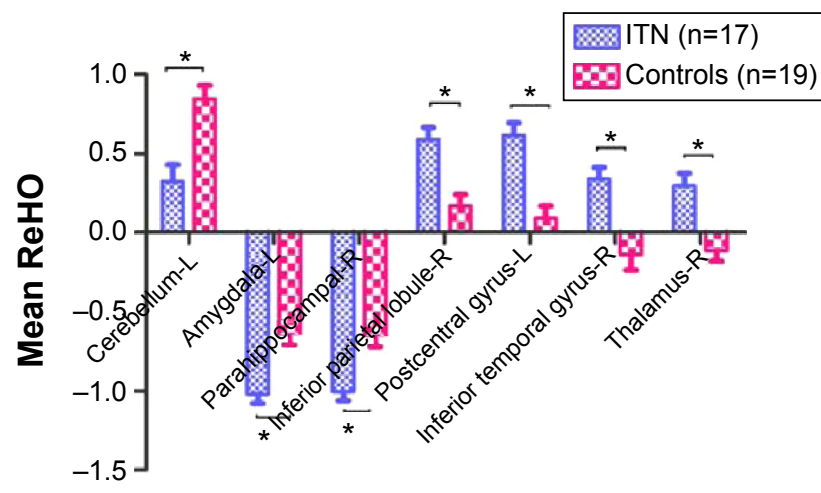

Significant clusters
C

Correlationship

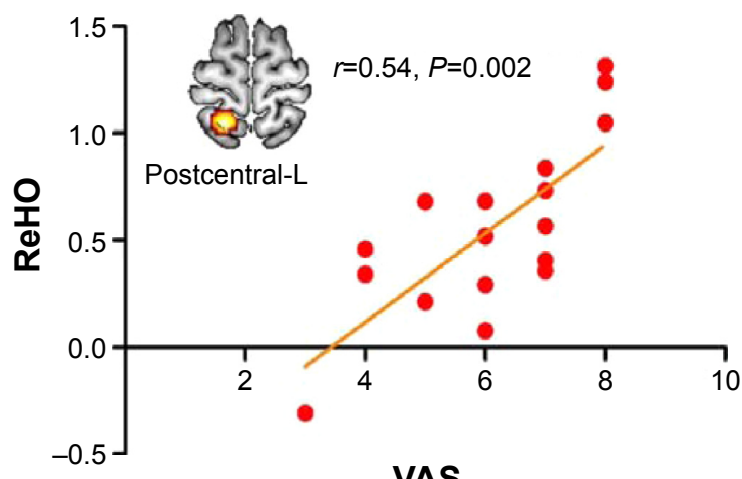

VAS

Figure I Group comparison of ReHo and its correlation with clinical variables.

Notes: (A) Voxel-wise comparison of ReHo. Group differences in ReHo between the ITN group and the control group (voxel-level $P<0.01$, cluster-level $P<0.05$, GRF correction). Blue-green colors indicate the ITN patients who had reduced ReHo while red-yellow indicates the ITN patients who had increased ReHo compared to the controls. (B) Plots of the significant clusters. The mean and standard deviation of ReHo values in significant clusters. $* P<0.05$. (C) Correlations between ReHo and clinical variables. A positive correlation between the ReHo increase in the left sensorimotor areas and pain intensity in ITN patients, ie, the higher the pain intensity the ITN patient, the greater the ReHo $(r=0.54, P=0.002)$.

Abbreviations: L, left; R, right; GRF, Gaussian random field; ITN, idiopathic trigeminal neuralgia; ReHo, regional homogeneity; VAS, visual analog scale.

In our study, altered patterns of ReHo were detected in patients with ITN, including decreased ReHo in the left amygdala, right parahippocampal gyrus, and left cerebellum and increased ReHo in the right inferior temporal gyrus, right thalamus, right inferior parietal lobule, and left postcentral gyrus. This is consistent with several structural brain imaging studies that have reported gray matter changes in pain perception, pain modulation, and motor areas. ${ }^{14,16}$ For example, reduced gray matter size was documented in the somatosensory cortex, thalamus, parahippocampus, and temporal lobe in ITN patients..$^{30}$ Another study ${ }^{14}$ reported greater gray matter volume in the thalamus, amygdala, periaqueductal gray matter, and basal ganglia. By contrast, ITN patients had thinner cortex in the anterior cingulate, the insula, and the orbitofrontal areas. Although inconsistency does exist in morphometric studies, the involved regions may also suggest a possible neuropathology in ITN.

The amygdala is a complex and deep brain structure involved in emotional processing. Fear conditioning and adjustment are the most noted emotional processes related to the amygdala,, 31 although its functional role has been extended to multiple dimensions including reward learning and motivation. ${ }^{9}$ Additionally, amygdala has been associated with a variety of psychiatric disorders, including anxiety ${ }^{32}$ and depression. ${ }^{33}$ Decreased ReHo in the left amygdala and parahippocampus is also reported in patients with experimentally induced lower back pain. ${ }^{34}$ Based on an investigation of the neurobiological significance of ReHo, ${ }^{21}$ decreased ReHo in the amygdala may indicate reduced functional integration of integrative information processing.

We also found that ITN patients had increased ReHo in the thalamus compared to controls. This result is consistent with a recent metabolic MRI study on ITN patients that showed a lower $\mathrm{N}$-acetylaspartate/creatine ratio in the affected-side thalamus and a negative relationship with VAS and disease duration. ${ }^{17}$ In addition, previous studies of ITN have reported greater patient thalamic volume relative to controls. ${ }^{14}$ It is reasonable to assume that the increased local synchronization of blood oxygenation level dependent (BOLD) time series 
in the thalamus reflects sustained nociceptive input, fitting with trigeminal neuropathy symptomology.

Both the parahippocampal gyrus and the lateral parietal cortex are regions of the default mode network (DMN), ${ }^{35}$ which is engaged in the internal processing including autobiographical memory, self-reference, and stimulus-independent thought. ${ }^{35-37}$ The DMN is generally active when it is not engaged with the external environment. ${ }^{35}$ The parahippocampus is involved in multiple behaviors, including stress and anxiety and the integration of complex information. ${ }^{38}$ Decreased parahippocampal ReHo in ITN patients supports its integrative role in internal processing and suggests that ITN involves decreased neural synchronization during internally directed or stimulus-independent mental states, which may be associated with an increased awareness of external nociceptive pain stimuli and anticipation. However, we also observed an increase in ReHo in the right lateral parietal cortex, and because they both constitute parts of DMN, the disparity between the parahippocampal decrease and the lateral parietal increase in ReHo could reflect maladaptive brain activity that is induced by pain. The lateral parietal cortex has been reported to participate in pain anticipation via meta-analysis, ${ }^{8}$ and it may represent a second level of the pain information processing circuit that supports the active, conscious, cognitive evaluation of pain sensation.

We also found increased ReHo in brain regions that generally encodes the discriminative properties of pain, including the pre- and postcentral gyri. The postcentral gyrus has been involved in the anticipation, intensity, discriminative, spatial and temporal summation aspects of pain processing. ${ }^{39-41}$ In patients with ITN, even simply non-painful movements can elicit attacks of pain, so restriction of facial movements would therefore ease the pain. The precentral gyrus (primary motor cortex) could therefore mirror sensory pain responses to repeated trigeminal neuralgia, motor inhibition of the maxilla and facial muscle tension. ${ }^{40}$ In our study, a positive relationship between pain intensity and ReHo in the left pre- and postcentral gyrus was found, suggesting a link between local synchronization of intrinsic brain activity and pain modulation.

It is also imperative to speculate whether altered $\mathrm{ReHo}$ occurs as an epiphenomenon that is secondary to daily stimulation or represents a functional predisposition to ITN in the light of peripheral vascular compression. ${ }^{15}$ However, our current study did not identify cingulate and insular involvement, which has been frequently reported in studies of pain anticipation $^{8,42}$ and investigation of the interplay between intrinsic brain networks. Our data directly link ratings of self-reported pain to local homogeneity of spontaneous brain activity in the sensorimotor areas, which may reflect the maladaptive process of daily pain attacks, while other ReHo changes across distributed brain regions may at least indicate a central role for the pathophysiology of ITN. Broadly, our findings have implications for understanding how ITN affects local intrinsic brain activity.

\section{Limitations}

The current analysis was restricted to local brain activity (local synchronization of resting BOLD signals). Consequently, only changes in regional connectivity could be detected, and it was not possible to determine how ITN affected the interactions among brain networks systems, which are more widely used. Studies of functional connectivity and topology could be used in the future to explore these questions. Furthermore, the patients in our study were not divided into subgroups, as described in Obermann et al. ${ }^{16}$ As such, we could not clarify a causal relationship for the pathophysiology of ITN. Last but not least, the functional MRI data were acquired on a 1.5 $\mathrm{T}$ scanner, negative influences for low-magnetic field MR in detecting between-group findings may exist.

\section{Conclusion}

In summary, our results provide novel evidence for the altered local synchronization of low-frequency spontaneous activity across central pain processing brain regions in patients with ITN. Furthermore, a positive relationship between pain intensity and ReHo in the sensorimotor areas was identified. This may reflect the maladaptive process of daily pain attacks, while other ReHo changes across distributed brain regions may indicate a central role for the pathophysiology of ITN. Broadly, our findings have implications for understanding how ITN affects local intrinsic brain activity.

These findings are in agreement with recent structural brain imaging results that have indicated that individuals with ITN have altered brain function involved in painprocessing ${ }^{15,16}$ and increased motor output, to control facial movements and to limit pain attacks. ${ }^{14}$

\section{Acknowledgments}

This work was supported by grants from Suzhou Science and Technology Development Program (SZS201205) and Suzhou Medical Key Discipline Project.

\section{Disclosure}

The authors report no conflicts of interest in this work.

\section{References}

1. Jannetta PJ. Observations on the etiology of trigeminal neuralgia, hemifacial spasm, acoustic nerve dysfunction and glossopharyngeal neuralgia. Definitive microsurgical treatment and results in 117 patients. Neurochirurgia (Stuttg). 1977;20(5):145-154. 
2. Kondo A. Follow-up results of microvascular decompression in trigeminal neuralgia and hemifacial spasm. Neurosurgery. 1997;40(1):46-51, 51-52.

3. Gronseth G, Cruccu G, Alksne J, et al. Practice parameter: the diagnostic evaluation and treatment of trigeminal neuralgia (an evidence-based review): report of the Quality Standards Subcommittee of the American Academy of Neurology and the European Federation of Neurological Societies. Neurology. 2008;71(15):1183-1190.

4. Katusic S, Williams DB, Beard CM, Bergstralh EJ, Kurland LT. Epidemiology and clinical features of idiopathic trigeminal neuralgia and glossopharyngeal neuralgia: similarities and differences, Rochester, Minnesota, 1945-1984. Neuroepidemiology. 1991;10(5-6):276-281.

5. Mueller D, Obermann M, Yoon MS, et al. Prevalence of trigeminal neuralgia and persistent idiopathic facial pain: a population-based study. Cephalalgia. 2011;31(15):1542-1548.

6. Obermann M, Yoon MS, Ese D, et al. Impaired trigeminal nociceptive processing in patients with trigeminal neuralgia. Neurology. 2007;69(9): 835-841.

7. Borsook D, Moulton EA, Pendse G, et al. Comparison of evoked vs. spontaneous tics in a patient with trigeminal neuralgia (tic doloureux) Mol Pain. 2007;3:34.

8. Palermo S, Benedetti F, Costa T, Amanzio M. Pain anticipation: an activation likelihood estimation meta-analysis of brain imaging studies. Hum Brain Mapp. 2015;36(5):1648-1661.

9. Simons LE, Moulton EA, Linnman C, Carpino E, Becerra L, Borsook D. The human amygdala and pain: evidence from neuroimaging. Hum Brain Mapp. 2014;35(2):527-538.

10. Kregel J, Meeus M, Malfliet A, et al. Structural and functional brain abnormalities in chronic low back pain: a systematic review. Semin Arthritis Rheum. 2015;45(2):229-237.

11. Nakata H, Sakamoto K, Kakigi R. Meditation reduces pain-related neural activity in the anterior cingulate cortex, insula, secondary somatosensory cortex, and thalamus. Front Psychol. 2014;5:1489.

12. Kucyi A, Davis KD. The dynamic pain connectome. Trends Neurosci. 2015;38(2):86-95.

13. DeSouza DD, Davis KD, Hodaie M. Reversal of insular and microstructural nerve abnormalities following effective surgical treatment for trigeminal neuralgia. Pain. 2015;156(6):1112-1123.

14. Desouza DD, Moayedi M, Chen DQ, Davis KD, Hodaie M. Sensorimotor and pain modulation brain abnormalities in trigeminal neuralgia: a paroxysmal, sensory-triggered neuropathic pain. PLoS One. 2013;8(6):e66340.

15. Parise M, Kubo TT, Doring TM, Tukamoto G, Vincent M, Gasparetto EL. Cuneus and fusiform cortices thickness is reduced in trigeminal neuralgia. J Headache Pain. 2014;15:17.

16. Obermann M, Rodriguez-Raecke R, Naegel S, et al. Gray matter volume reduction reflects chronic pain in trigeminal neuralgia. Neuroimage. 2013;74:352-358.

17. Wang Y, Li D, Bao F, et al. Thalamic metabolic alterations with cognitive dysfunction in idiopathic trigeminal neuralgia: a multivoxel spectroscopy study. Neuroradiology. 2014;56(8):685-693.

18. Gu T, Ma XX, Xu YH, Xiu JJ, Li CF. Metabolite concentration ratios in thalami of patients with migraine and trigeminal neuralgia measured with 1H-MRS. Neurol Res. 2008;30(3):229-233.

19. Zang Y, Jiang T, Lu Y, He Y, Tian L. Regional homogeneity approach to fMRI data analysis. Neuroimage. 2004;22(1):394-400.

20. Zuo XN, Xu T, Jiang L, et al. Toward reliable characterization of functional homogeneity in the human brain: preprocessing, scan duration, imaging resolution and computational space. Neuroimage. 2013; 65:374-386.

21. Jiang L, Xu T, He Y, et al. Toward neurobiological characterization of functional homogeneity in the human cortex: regional variation, morphological association and functional covariance network organization. Brain Struct Funct. 2015;220(5):2485-2507.
22. Benoliel R, Eliav E. Neuropathic orofacial pain. Alpha Omegan. 2012; 105(3-4):66-74

23. Beck AT, Ward C, Mendelson M. Beck depression inventory (BDI). Arch Gen Psychiatry. 1961;4(6):561-571.

24. Hamilton M. The assessment of anxiety states by rating. $\mathrm{Br} \mathrm{J} \mathrm{Med}$ Psychol. 1959;32(1):50-55.

25. Ashburner J. A fast diffeomorphic image registration algorithm. Neuroimage. 2007;38(1):95-113.

26. Liu D, Yan C, Ren J, Yao L, Kiviniemi VJ, Zang Y. Using coherence to measure regional homogeneity of resting-state FMRI signal. Front Syst Neurosci. 2010;4:24.

27. Fox SS, Norman RJ. Functional congruence: an index of neural homogeneity and a new measure of brain activity. Science. 1968; 159(3820):1257-1259.

28. Bullmore E, Sporns O. Complex brain networks: graph theoretical analysis of structural and functional systems. Nat Rev Neurosci. 2009;10(3): 186-198.

29. Jiang L, Zuo X. Regional homogeneity a multimodal, multiscale neuroimaging marker of the human connectome. Neuroscientist. Epub 2015 Jul 13. doi:10.1177/1073858415595004.

30. Obermann M, Rodriguez-Raecke R, Naegel S, et al. Gray matter volume reduction reflects chronic pain in trigeminal neuralgia. Neuroimage. 2013; $74: 352-358$

31. LeDoux JE. Emotion circuits in the brain. Annu Rev Neurosci. 2000;23: 155-184.

32. Etkin A, Wager TD. Functional neuroimaging of anxiety: a metaanalysis of emotional processing in PTSD, social anxiety disorder, and specific phobia. Am J Psychiatry. 2007;164(10):1476-1488.

33. Sacher J, Neumann J, Funfstuck T, Soliman A, Villinger A, Schroeter ML. Mapping the depressed brain: a meta-analysis of structural and functional alterations in major depressive disorder. $J$ Affect Disord. 2012;140(2):142-148.

34. Zhang SS, Wu W, Liu ZP, Huang GZ, Guo SG, Yang JM. Altered regional homogeneity in experimentally induced low back pain: a resting-state fMRI study. J Neuroeng Rehabil. 2014;11:115.

35. Raichle ME, MacLeod AM, Snyder AZ, Powers WJ, Gusnard DA, Shulman GL. A default mode of brain function. Proc Natl Acad Sci US A. 2001;98(2):676-682.

36. Buckner RL, Andrews-Hanna JR, Schacter DL. The brain's default network: anatomy, function, and relevance to disease. Ann NY Acad Sci. 2008;1124:1-38.

37. Kucyi A, Moayedi M, Weissman-Fogel I, et al. Enhanced medial prefrontal-default mode network functional connectivity in chronic pain and its association with pain rumination. JNeurosci. 2014;34(11): 3969-3975.

38. Sauro KM, Becker WJ. The stress and migraine interaction. Headache. 2009;49(9):1378-1386.

39. Apkarian AV, Bushnell MC, Treede RD, Zubieta JK. Human brain mechanisms of pain perception and regulation in health and disease. Eur J Pain. 2005;9(4):463-484.

40. Ellingson LD, Shields MR, Stegner AJ, Cook DB. Physical activity, sustained sedentary behavior, and pain modulation in women with fibromyalgia. J Pain. 2012;13(2):195-206.

41. Bantick SJ, Wise RG, Ploghaus A, Clare S, Smith SM, Tracey I. Imaging how attention modulates pain in humans using functional MRI. Brain. 2002;125(Pt 2):310-319.

42. Laird AR, Fox PM, Price CJ, et al. ALE meta-analysis: controlling the false discovery rate and performing statistical contrasts. Hum Brain Mapp. 2005;25(1):155-164. 


\section{Publish your work in this journal}

Neuropsychiatric Disease and Treatment is an international, peerreviewed journal of clinical therapeutics and pharmacology focusing on concise rapid reporting of clinical or pre-clinical studies on a range of neuropsychiatric and neurological disorders. This journal is indexed on PubMed Central, the 'PsycINFO' database and CAS,

and is the official journal of The International Neuropsychiatric Association (INA). The manuscript management system is completely online and includes a very quick and fair peer-review system, which is all easy to use. Visit http://www.dovepress.com/testimonials.php to read real quotes from published authors.

Submit your manuscript here: http://www.dovepress.com/neuropsychiatric-disease-and-treatment-journal 